\title{
Critical Point and Deconfinement in Stochastic Thermal Fields
}

\author{
G. Kozlov* \\ Joint Institute for Nuclear Research \\ E-mail: kozlov@jinr.ru
}

\begin{abstract}
The critical point in particle physics at high temperature is studied through the stochastic gas of scalars, the dilatons, in the model that implies the spontaneous breaking of an approximate scale symmetry. We found the critical temperature as a function of a dilaton mass, and the fluctuation of particle density grows up very sharply at critical point. Our results also suggest that the critical point may be identified through the fluctuation in yield of primary direct photons induced by conformal anomaly of strong and electromagnetic sectors.
\end{abstract}

The 36th Annual International Symposium on Lattice Field Theory - LATTICE2018

22-28 July, 2018

Michigan State University, East Lansing, Michigan, USA.

\footnotetext{
${ }^{*}$ Speaker.
} 


\section{Introduction}

The spontaneous breaking of symmetry has a major role in particle physics and cosmology where the phase transitions (PT) can occur at extreme conditions (e.g., high enough temperature, baryonic density, chemical baryonic potential). The critical point (CP) of PT corresponds to an initial thermal state (of matter) which is invariant under the conformal group. In the early Universe, the light massive and/or massless states are emerged. The critical phenomena if occurred are considered here through quantum PT with Bose-Einstein condensation (BEC) of the scalar stochastic field in a single zero mode of an almost ideal Bose-gas that suggests the breaking of conformal symmetry.

Quantum effects, e.g. gluon fluctuations, break conformal (scale) invariance. It is seen through the anomaly in the trace of energy-momentum tensor $\theta_{\mu}^{\mu}=\partial_{\mu} S^{\mu} \neq 0$, where the dilatation current $S^{\mu}=\theta^{\mu v} x_{v}$ does not conserved itself with respect to the scale transformations of coordinates $x_{\mu} \rightarrow \omega x_{\mu}$ ( $\omega$ is an arbitrary constant). The dilaton appeared as a pseudo-Goldstone boson is associated with the chiral condensate occurred in the region where the gauge coupling constants are slowly running and an effective fermion coupling constant does reach the critical value [1]. Because of the presence of strong gluon fields, the QCD vacuum is disordered and scale invariance is destroyed by the appearance of the dimensional scale $M=M_{U V} \exp \left[-8 \pi^{2} /\left(b_{0} g^{2}\right)\right]$, with $M_{U V}$ being the ultra-violet (UV) scale, $g$ is the bare gauge coupling constant and $b_{0}$ is the first coefficient in the QCD $\beta$-function, $\beta(M) \neq 0$. The breaking of conformal invariance assumes that all the processes are governed by the conformal anomaly (CA) resulting from running coupling constant $g(M)$ in $\beta$-function related to divergence of $S_{\mu}$. From this, $S_{\mu}$ is already non-conserved in the theory containing, e.g., the gluon and quark degrees of freedom (d.o.f.). The divergence of $S_{\mu}$ is proportional to $\beta$-function and the quark masses. There could be an approximate scale (dilatation) symmetry if $\beta(g)$ is small enough and $g$ is slowly running with $M$. Theory becomes conformal in the infra-red (IR) with the non-trivial solution $\alpha_{s}^{\star}=-2 \pi b_{0} / b_{1}$ (IR fixed point (IRFP)) in the perturbative domain if $b_{0}=\left(11 N_{c}-2 N_{f}\right) / 3$ is small $\left(N_{c}\right.$ and $N_{f}$ are the numbers of colors and flavors, respectively). The dilatons are unstable, they decay into two photons, where $\mathrm{CA}$ acts as a source of primary direct photons. The signature of $\mathrm{CP}$ is non-monotonous behavior of observable fluctuation where the latter increases very crucially. The dilaton is a messenger (mediator) between conformal sector and the Standard Model (SM). However, at high enough temperatures the conformal sector has no direct couplings to SM because of absence of CA $\left(\theta_{\mu}^{\mu}=0\right)$. We suggest the novel approach to an approximate scale symmetry breaking with the challenge phenomenology where the primary direct photons induced by $\mathrm{CA}$ are in fluctuating regime The latter is an indicator of $\mathrm{CP}$ if the correlation length grows to become very large.

\section{Dilatons through quantum statistical states}

We start with the dilaton-pion model given by the Lagrangian density (LD)

$$
L=\frac{1}{2} f_{\sigma}^{2}\left(\partial_{\mu} e^{\sigma}\right)^{2}+\frac{1}{2} f_{\pi}^{2} e^{2 \sigma} \partial_{\mu} \pi+\ldots
$$


which is scale invariant under transformations of coordinates $x_{\mu} \rightarrow \omega x_{\mu}$, if $\pi(x)$ transforms as $\pi(x) \rightarrow \pi\left(x e^{\omega}\right)$ and the dilaton field $\sigma$ transforms non-linearly

$$
\sigma(x) \rightarrow \sigma\left(x e^{\omega}\right)+\omega
$$

$\operatorname{LD}(2.1)$ has the more suitable form $L=(\partial \chi)^{2} / 2+\left(f_{\pi} / f_{\chi}\right)^{2} \chi^{2} \partial \pi+\ldots$, if one makes redefinition of the dilaton field $\sigma(x) \rightarrow \chi(x)=f_{\chi} e^{\sigma(x)}$, which transforms non-linearly under (2.2), $\langle\chi\rangle=f_{\chi} . S_{\mu}$ acting on the vacuum $|0\rangle$ defines $\chi:\left\langle 0\left|S^{\mu}\right| \chi(p)\right\rangle=i p^{\mu} f_{\chi}, \partial_{\mu}\left\langle 0\left|S^{\mu}(x)\right| \chi(p)\right\rangle=\left\langle 0\left|\theta_{\mu}^{\mu}(x)\right| \chi(p)\right\rangle=$ $-f_{\chi} m_{\chi}^{2} e^{-i p x}$, where for on-shell case $\left\langle 0\left|\theta^{\mu v}(x)\right| \chi(p)\right\rangle=f_{\chi}\left(p^{\mu} p^{v}-g^{\mu v} p^{2}\right) e^{-i p x}, p^{2}=m_{\chi}^{2}$. Here, $m_{\chi}$ is the mass of the dilaton, $p_{\mu}$ is the momentum conjugate to $x_{\mu} . f_{\chi}$ is the decay constant of a dilaton. We suppose that at the scale larger than that of the confinement scale $\Lambda$ the dilaton is formed as the bound state of two gluons, the glueball $\chi=O^{++}$, with the mass $m_{\chi} \sim O(\Lambda)$. The characteristic feature of the $\mathrm{CP}$ is sharp increasing of the fluctuations of the order parameter field $\chi$. They act as a regulator in the IR with correlation length $\xi=m_{\chi}^{-1}$. The latter is not measured directly, however, it influences the fluctuations of observed particles (observables), e.g., the primary photons to which the critical mode couples. In the vicinity of $\mathrm{CP}, \xi$ is much higher that that of a size of the particle interacting region at early times.

Consider the system containing dilatons as almost ideal weakly interacting stochastic gas at finite temperature. It may correspond to the state of almost quark-gluon plasma in terms of ideal liquid with minimal viscosity. In the state of statistical equilibrium at temperature $T=\beta^{-1}$ the partition function for $N$ particles is

$$
Z_{N}=S p e^{-H \beta},
$$

where $H$ is the Hamiltonian $H=\sum_{1 \leq j \leq N} H(j)$, and $\beta$ in (2.3) differs from those of the QCD $\beta$ - function. For the system of regular dilaton functions $\sigma_{f}(x)$ in $f$ representation one has the equation $H(j) \sigma_{f}\left(x_{j}\right)=F(f) \sigma_{f}\left(x_{j}\right)$, where $H=\sum_{f} F(f) b_{f}^{+} b_{f}=\sum_{f} F(f) n_{f}$ in terms of operators of creation $b_{f}^{+}$and annihilation $b_{f} ; n_{f}$ is an occupation (particle) number. Here, $F(f)=E(f)-$ $\mu Q(f)$ with $E(f)$ being the energy, $\mu$ is the chemical potential, $Q(f)$ is the conserved charge with an average density

$$
\langle q\rangle=\frac{1}{\Omega}\langle Q\rangle=\frac{1}{\Omega} \frac{1}{\beta} \frac{\partial}{\partial \mu} \ln Z_{N},
$$

$\Omega$ is the volume of a system. In quantum statistical mechanics where the open system has a thermal contact and a particle interaction with a reservour, $Q$ is an operator $N_{f}$ of particles of the type $f$ with the mean value $\operatorname{Tr}\left\{\rho N_{f}\right\}=\hat{n}_{f} \Omega$, where $\rho$ is the statistical operator, $\hat{n}_{f}$ is the density of particles of the type $f$. Interactions between glueballs should lead to thermal equlibrium, and in case of large $n_{f}$ to the formation of BEC. In principle, operators $b_{f}$ can be distorted by random quantum stochastic fluctuations through the stochastic operator $r_{f}, b_{f} \rightarrow b_{f}=a_{f}+r_{f}$, where $a_{f}$ is the bare (annihilation) operator. The function $Z_{N}$ (2.3) has the form [2]

$$
Z_{N}=\sum_{\ldots n_{f} \ldots, \sum_{f} n_{f}=N} e^{-\beta \sum_{f} F(f) n_{f}} .
$$

Since all the operators ... $n_{f} .$. commute to each other, they may be clarified through the observables. The calculation of (2.4) meets difficulties because of the condition $\sum_{f} n_{f}=N$ for fixed $N$ and within the limit $N \rightarrow \infty$ in the final stage calculations. The latter condition is important because of particle 
decays: the glueballs are unstable, hence they may decay into two primary direct photons (not produced from hadron decays) which are registered as a signal that the vicinity of CP is approached. The phase transitions are characterised through the singularities (discontinuities) in the dependence of various observables on the parameters, e.g., temperature, chemical potential etc. On the plain of the phase diagram, CP manifests itself through the critical chemical potential $\mu_{c}$ and the critical temperature $T_{c}$. Let us consider the following power series

$$
P(\bar{\mu})=\sum_{N=1}^{\infty} Z_{N} \bar{\mu}^{N}
$$

which is the scan function on $\mu$ and $\beta$, where $\bar{\mu}=\mu / \mu_{c}$. Having in mind (2.4) one has

$$
P(\bar{\mu})=\sum_{\ldots n_{f} \ldots} e^{-\beta \sum_{f} F(f) n_{f}} \bar{\mu}^{\Sigma_{f} n_{f}}=\prod_{f}\left[\sum_{0 \leq n<\infty} e^{-\beta F(f) n} \bar{\mu}^{n}\right]=\prod_{f} \frac{1}{1-\bar{\mu} e^{-F(f) \beta}} .
$$

Let us consider for simplicity that $F(f) \geq 0$ in (2.6). Actually, the convergence radius $R$ of the series (2.5) will not be less than 1. In the vicinity of $\mathrm{CP}(\bar{\mu} \simeq 1)$ one has $\mu_{c}<E(f) / Q(f)$, that is trivial because of the dilaton mass $m_{\chi} \rightarrow 0$ if $E=|\vec{p}|^{2} /\left(2 m_{\chi}\right)$, where $|\vec{p}|$ is the momentum of $\chi$.

Let us consider (2.5) in the form

$$
\frac{P(\bar{\mu})}{\bar{\mu}^{N}}=\sum_{N^{\prime}=0}^{\infty} \frac{Z_{N^{\prime}} \bar{\mu}^{N^{\prime}}}{\bar{\mu}^{N}}
$$

on the real axis $0<\bar{\mu}<R$. Because of positive $Z_{N^{\prime}}$ the scan function (2.7) has the only one minimum on $(0, R)$

$$
\frac{d^{2}}{d \bar{\mu}^{2}}\left[P(\bar{\mu}) \bar{\mu}^{-N}\right]=\sum_{N^{\prime}=0}^{\infty}\left(N^{\prime}-N\right)\left(N^{\prime}-N-1\right) Z_{N^{\prime}} \bar{\mu}^{N^{\prime}-N-2}>0 .
$$

The function (2.7) tends to infinity when $\bar{\mu} \rightarrow 0$ and when $\bar{\mu} \rightarrow R$. In the interval $(0, R)$ there is a point $\bar{\mu}=\bar{\mu}_{0}$ at which (2.7) has a single minimum, i.e.

$$
\frac{d}{d \bar{\mu}}\left[P(\bar{\mu}) \bar{\mu}^{-N}\right]_{\mid \bar{\mu}=\bar{\mu}_{0}}=\sum_{N^{\prime}=0}^{\infty} Z_{N^{\prime}}\left(N^{\prime}-N\right) \bar{\mu}_{\mid \bar{\mu}=\bar{\mu}_{0}}^{N^{\prime}-N-1}=0 .
$$

If one goes alone the vertical axis, (2.7) has a maximum at $\bar{\mu}_{0}$. As long as $\bar{\mu}<\bar{\mu}_{0}$ no state with $Q \neq 0$ can compete with the vacuum state $(E=0, Q=0)$ for the role of the ground state. In case when $\bar{\mu}>\bar{\mu}_{0}$, the point $\bar{\mu}=\bar{\mu}_{0}$ is the ground state at given $\mu$. In the phase space the spectrum of "quasimomenta" $f$ is almost continuous, and there will be an exact continuos spectrum in the limit $\Omega \rightarrow \infty$. The number $\Delta N$ of different $\Delta f$ in volume $\Omega$ is $(\Delta N / \Delta f)=$ const.$\Omega$. Having in mind that

$$
P(\bar{\mu})=\exp \left\{-\sum_{f} \ln \left[1-\bar{\mu} e^{-F(f) \beta}\right]\right\}
$$

one can find the asymptotic equality $\sum_{f} \ln \left[1-\bar{\mu} e^{-F(f) \beta}\right]=N \Phi(\bar{\mu})$, where $\Phi(\bar{\mu})=\operatorname{const} \cdot v \beta K_{\chi}(\bar{\mu})$, $v=\Omega / N . K_{\chi}(\bar{\mu})$ is the thermochemical potential of the dilaton $\chi$

$$
K_{\chi}(\bar{\mu})=\beta^{-1} \int \ln \left[1-\bar{\mu} e^{-F(f) \beta}\right] d f,
$$


which gives the contribution to thermodynamic potential $K=K_{\chi}+V_{\chi}+\lambda\left(f_{\chi} / 2\right)^{4}$. Here, $V_{\chi}$ is the potential term in LD of the dilaton $L_{\chi}=(1 / 2) \partial_{\mu} \chi \partial^{\mu} \chi-V_{\chi}, V_{\chi}=(\lambda / 4) \chi^{4}\left[\ln \left(\chi / f_{\chi}\right)-1 / 4\right]$. The term $\lambda\left(f_{\chi} / 2\right)^{4}$ in $K$ is added so that $K=0$ at $T=0$ and $\chi=f_{\chi}$. In the vicinity of CP, the free gluons are disfavored as appropriate degrees of freedom in the phase with confinement of quarks. The thermodynamic potential $K$ does account for dilaton (glueball) and gluon degrees of freedom: $K=\theta\left(\beta_{c}-\beta\right) K_{\chi}(\bar{\mu})+\theta\left(\beta-\beta_{c}\right) K_{g}$, where $K_{g}$ is an effective gluon thermodynamic potential with the energy $E_{g}=\sqrt{|\vec{p}|^{2}+m_{g}^{2}}, m_{g}$ is an effective gluon mass. $K_{g}$ is model-dependent function and we propose tfhe following its form

$$
K_{g}=\beta^{-1} \int \ln \left[1-e^{-E_{g}(f) \beta}\right] d f .
$$

Both forms (2.8) and (2.9) match to each other at CP. The ground state $\bar{\mu}_{0}$ is defined from the Eq.

$$
\sum_{f} \bar{n}_{f}=\sum_{f} \frac{1}{\bar{\mu}_{0}^{-1} e^{F(f) \beta-1}}=N .
$$

The infinite number of particles $N$ is expected if $\bar{\mu}_{0}=1$ and $F(f)=0$. Hence, $\bar{\mu}_{0}$ may be associated with CP. The large number $N$ is correct if the dilatons are light. This is important in the sense of the proposal to condensed dark matter bosons in the early stage after (heavy ion) collisions. The latter in some sense corresponds to Bose star formation as the lamps of Bose-Einstein condensate bounded by self-gravity [3].

\section{A vicinity of the critical point}

Consider the nonrelativistic model where the glueballs are produced in the volume $\Omega$ as a cube with the side of the length $L=\Omega^{1 / 3}$. In the limit $\Omega \rightarrow \infty$ and for $v=$ const we consider two cases: high temperature case A), where $\bar{\mu}_{0} e^{\mu Q \beta}<1$, and low $T$ case B), where $\bar{\mu}_{0} e^{\mu Q \beta} \sim 1$. In case A) the function $\bar{n}_{f}$ is regular on $f$, and the sum $\sum_{f} \bar{n}_{f}$ is replaced by the integral, where the spectrum of $f$ is continuous at $\Omega \rightarrow \infty$ :

$$
\frac{1}{v}=\frac{1}{\Omega} \sum_{f} \bar{n}_{f} \rightarrow \frac{1}{(2 \pi)^{3}} \int \bar{n}(f) d^{3} f
$$

Using the form of $\bar{n}(f)(2.10)$ we have an equality:

$$
\int_{0}^{\infty} \frac{x^{2} d x}{\bar{\mu}_{0}^{-1} e^{-\mu Q \beta} e^{x^{2}}-1}=\frac{2 \pi^{2}}{v}\left(\frac{\beta}{2 m_{\chi}}\right)^{3 / 2} .
$$

The integral in 1.h.s. of (3.1) increases if $\mu Q+T \ln \bar{\mu}_{0} \rightarrow 0$ that will allow one to find an unequality

$$
\frac{2 \pi^{2}}{v}\left(\frac{\beta}{2 m_{\chi}}\right)^{3 / 2}<\int_{0}^{\infty} \frac{x^{2} d x}{e^{x^{2}}-1}
$$

The case A) is realized when $T>T_{c}$, where

$$
T_{c}=\frac{1}{2 m_{\chi}}\left(\frac{2 \pi^{2}}{v B}\right)^{2 / 3}, B=\frac{\sqrt{\pi}}{4} \cdot 2,612 \ldots, m_{\chi} \neq 0 .
$$


One can easily find the singular behavior of the correlation length $\xi$, where divergence of $\xi$ is governed by the ground state $\bar{\mu}_{0}$ :

$$
\xi=2 \mu Q\left(\frac{v B}{2 \pi^{2}}\right)^{2 / 3} \ln ^{-1}\left(\frac{1}{\bar{\mu}_{0}}\right) .
$$

Actually, $\xi \rightarrow \infty$ at $\bar{\mu}_{0} \rightarrow 1$ that means $\mathrm{CP}\left(\bar{\mu}_{0}=\bar{\mu}=1\right)$.

In case $\mathrm{B}$ ) our interest is in small $|p| \leq \delta$ (maximal $N$ ), where $\mathrm{CP}$ is approached. Here

$$
\frac{1}{\Omega} \sum_{|p| \leq \delta} \bar{n}_{p}=\frac{1}{v}-\frac{1}{\Omega} \sum_{|p| \geq \delta} \bar{n}_{p}
$$

where

$$
\lim _{\delta \rightarrow 0, N \rightarrow \infty} \frac{1}{\Omega} \sum_{|p| \leq \delta} \bar{n}_{p}=\frac{1}{v}-\frac{1}{(2 \pi)^{3}} \int_{|p| \geq \delta} \frac{d^{3} p}{e^{E_{p} \beta}-1}=\frac{1}{v}\left[1-\left(\frac{\beta_{c}}{\beta}\right)^{3 / 2}\right] .
$$

In (3.3) the only part of total number of particles proportional $\sim\left(\beta_{c} / \beta\right)^{3 / 2}$ is distributed on all the spectrum of momenta. The rest one $\sim\left[1-\left(\beta_{c} / \beta\right)^{3 / 2}\right]$ is the scalar condensate. The fluctuation of particle density in volume $V$, which is (much) less than $\Omega$, is the function of $\xi$

$$
\left\langle\left(n_{V}-\left\langle n_{V}\right\rangle\right)^{2}\right\rangle=\left\langle n_{V}\right\rangle\left[1+\frac{\sqrt{2} v}{\pi^{2}}\left(\frac{T}{\xi}\right)^{3 / 2} \int_{0}^{\infty} \frac{x^{2} d x}{\left(\bar{\mu}_{0}^{-1} e^{-\mu Q \beta} e^{x^{2}}-1\right)^{2}}\right],\left\langle n_{V}\right\rangle=V / \Omega .
$$

The sharp increasing of (3.4) is expected at CP, and it is $\xi$-independent.

\section{Direct photons at $\mathrm{CP}$}

In the exact scale symmetry, $\chi$ couples to SM particles through the trace of $\theta_{\mu \nu}$

$$
L=\frac{\chi}{f_{\chi}}\left(\theta_{\mu_{\text {tree }}}^{\mu}+\theta_{\mu_{\text {anom }}}^{\mu}\right),
$$

where the first term is (contributions from heavy quarks and heavy gauge bosons are neglected)

$$
\theta_{\mu_{\text {tree }}}^{\mu}=-\sum_{q}\left[m_{q}+\gamma_{m}(g)\right] \bar{q} q-\frac{1}{2} m_{\chi}^{2} \chi^{2}+\partial_{\mu} \chi \partial^{\mu} \chi
$$

$q$ is a quark d.o.f. with the mass $m_{q}, \gamma_{m}$ are the corresponding anomalous dimensions. In contrast to SM, the dilaton couples to massless gauge bosons even before running any SM particles in the loop, through the trace anomaly. The latter has the following term in (4.1) for photons and gluons:

$$
\theta_{\mu_{\text {anom }}}^{\mu}=-\frac{\alpha}{8 \pi} b_{E M} F_{\mu v} F^{\mu v}-\frac{\alpha_{s}}{8 \pi} \sum_{i} b_{0_{i}} G_{\mu \nu}^{a} G^{\mu v a}
$$

where $\alpha$ is the fine coupling constant, $b_{E M}$ and $b_{0_{i}}$ are the coefficients of electromagnetic (EM) and QCD $\beta$-functions, respectively. If the strong (and EM) interactions are embedded in the conformal sector the following relation for light and heavy particles sectors is established above the scale 
$\Lambda: \sum_{\text {light }} b_{0}=-\sum_{\text {heavy }} b_{0}$, where the mass of $\chi$ splits the light and heavy states. The anomaly (non-perturbative) term for gluons in (4.1)

$$
\frac{\alpha_{s}}{8 \pi} b_{0}^{\text {light }} G_{\mu \nu}^{a} G^{\mu v a}=\frac{\beta(g)}{2 g} G_{\mu \nu}^{a} G^{\mu v a}, b_{0}^{\text {light }}=-11+\frac{2}{3} n_{L}
$$

is evident, where the only $n_{L}$ particles, lighter than $\chi$, are included in the $\beta$ - function, $\beta(g)=$ $b_{0}^{\text {light }} g^{3} /\left(16 \pi^{2}\right)$. For $m_{\chi} \sim O(\Lambda)$ one has $n_{L}=3$ that indicates about 14 times increase of the dilaton-gluon-gluon coupling strength compared to that of the SM Higgs boson. The partial decay width $\chi \rightarrow \gamma \gamma$ is

$$
\Gamma(\chi \rightarrow \gamma \gamma) \simeq\left(\frac{\alpha F_{\text {anom }}}{4 \pi}\right)^{2} \frac{m_{\chi}^{3}}{16 \pi f_{\chi}^{2}}
$$

where the only CA does contribute through $F_{\text {anom }}=-\left(2 n_{L} / 3\right)\left(b_{E M} / b_{0}^{\text {light }}\right), b_{0}^{\text {light }}=-11+(2 / 3) n_{L}$, $b_{E M}=-4 \sum_{q: u, d, s} e_{q}^{2}=-8 / 3, e_{q}$ is the charge of the light quark. In the vicinity of IRFP, there are fluctuations of dilaton field with $m_{\chi} \simeq \sqrt{1-N_{f} / N_{f}^{c}} \Lambda$ [4], where $N_{f}^{c}$ is the critical value of $N_{f}$ corresponding to $\alpha_{s}^{c}$ at which the chiral symmetry is breaking and the confinement is emerged. When one approaches the CP, the absolute value of $F_{\text {anom }}$ decreases due to increasing of $b_{0}^{\text {light }}$ as $n_{L} \rightarrow 0$. The second-order phase transition is characterized by the limits $N_{f} \rightarrow N_{f}^{c}$ and $\Lambda \rightarrow 0$, hence no direct photons should be evident through a detector. In the IR one can estimate the fluctuation rate relevant to primary photons compared to two photons escape due to decay of neutral $\pi^{0}$ mesons, $\pi^{0} \rightarrow \gamma \gamma$ (at very large distances, the effective d.o.f. are pions):

$$
r_{\chi} \sim \Gamma\left(\pi^{0} \rightarrow \gamma \gamma\right)\left(\Lambda / n_{L}\right)^{2} \xi^{3},
$$

where $\xi$ is given by (3.2). Actually, $r_{\chi}$ (4.2) in terms of confinement scale $\Lambda$ is scheme independent. At CP, $r_{\chi} \rightarrow \infty$ when the number of light quarks $n_{L} \rightarrow 0$ as well as the fluctuation length $\xi$ is sharply increasing. The measurement of photon fluctuations can be used to determine whether the quantum system is in the vicinity of $\mathrm{CP}$ or not.

\section{Conclusion}

In the novel approach to an approximate scale symmetry breaking the $\mathrm{CP}$ is achieved at higher $\mu$, the case B), with smaller particle momentum (and, hence, the energy). In the vicinity of CP we find the the sharp increasing of particle density at $\mathrm{CP}$. There is non-monotonous behavior in fluctuations of direct photons which grow in IR to become large at CP. The location of CP for the given experimental conditions is obtained by measuring the ratios of $\gamma$-quanta yields and compared (fitting) to known model with $T$ and $\mu$.

\section{References}

[1] W.A. Bardeen, C.N. Leung, and S.T. Love, Phys. Rev. Lett. 56 (1986) 1230.

[2] N.N. Bogolyubov, Collection of Scientific Papers in 12 vol. Equilibrium Statistical Mechanics: Vol. 6, p. 80, 2005, Nauka, Moscow, ISBN 5-02-034457-5 [in Russian].

[3] D.G. Levkov, A.G. Panin, I.I. Tkachev, Phys. Rev. Lett. 121 (2018) 151301.

[4] T. Appelquist and Y. Bai, Phys. Rev. D82 (2010) 071701. 